\title{
Instrucción, aprendizaje e interacción profesor-alumno. Un estudio de observación en el aula ${ }^{1}$
}

\author{
MARÍA CRUZ García, Alberto Rosa, IGNACIO \\ MONTERO y ETIEDEM ${ }^{2}$
}

Universidad Autónoma de Madrid / Centro Nacional de Recursos para la Educación Especial

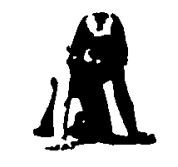

Resumen

Este trabajo aborda el estudio de la incidencia de los procesos de enseñanza en el aumento de conocimientos y destrezas de un grupo de niños paraliticos cerebrales en los primeros ciclos de enseñanza formal. Ello se hace a través de la puesta en relación de la ganancia que los sujetos manifiestan en sus rendimientos en una prueba criterial con datos de observaciones sobre las actividades realizadas en el aula. Los resultados muestran la existencia de un progreso en todas las áreas evaluadas, sin que parezca influir el nivel de afectación motora o del babla. Se observa que sólo menos de un tercio de las tareas escolares se plantean por encima del nivel de conocimientos previos del alumno, en estos casos la forma de interacción entre alumno y profesor toman caracteristicas peculiares que permiten caracterizar la forma de trabajo en la zona de desarrollo próximo.

Palabras clave: Instrucción-aprendizaje, Parálisis cerebral, Zona de desarrollo próximo.

\section{Instruction, learning and teacher-child interaction. An observational study in the classroom}

\section{Abstract}

This paper studies the effect of instruction on the development of school and cognitive skills in a group of cerebral palsied preschoolers and first and second graders. Pre-test and post-test measurements were taken prior to and after an instructional treatment lasting one academic year that was designed an carried out by teachers. Changes in performance were related to data gathered through observation in the classroom. Results show a progress in all the areas tested, factors such as the level of motor or verbal impairment do not seem to affect the degree of improvement on test performance. It was observed that less than one third of the instructional tasks performed in the classroom required a level of ability above that shown by students in the pre-test. Observational data offer information that may help to describe some characteristics of instructional work within the Zone of Proximal Development.

Key-words: Instruction, Learning, Cerebral palsy, Zone of proximal development.

Dirección de los autores: Centro Nacional de Recursos para la Educación Especial. General Oraa, 55. 28006 Madrid. Universidad Autónoma de Madrid, Facultad de Psicología. 28071 Madrid.

(C) 1990 by Aprendizaje

Infancia y Aprendizaje, 1990, 51-52, 79-97 


\section{INTRODUCCION}

El trabajo que aquí se presenta forma parte de una investigación más amplia cuyos resultados se irán haciendo públicos conforme vayan estando disponibles ${ }^{3}$. El objeto de este artículo es presentar los resultados de un estudio preliminar y descriptivo - que no tiene la intención de ser generalizable a la población- sobre la relación existente entre el progreso observado en un grupo de alumnos paralíticos cerebrales y el tratamiento educativo que han recibido por parte de sus profesores a lo largo de un curso académico. Nuestro intento es el realizar un estudio descriptivo, pero metodológicamente riguroso, de algunos aspectos del trabajo en el aula que suelen estudiarse de forma cualitativa. Quizá podría decirse que tratamos de utilizar una metodología "dura» para tratar temáticas que tradicionalmente vienen considerándose como «blandas».

El objetivo inicial de esta investigación era el estudiar las posibilidades de los microordenadores como apoyo al proceso de aprendizaje de los niños con deficiencias motóricas, centrado especialmente en la parálisis cerebral. Sin embargo, pronto se hizo evidente que este objetivo sólo podía ser abordado de una manera efectiva si el uso del ordenador se consideraba dentro del contexto de los procesos de instrucción y aprendizaje que se producen en la escuela y que tienen como sujeto a un individuo en desarrollo (ver Rosa, 1985). A ello hay que añadir que el enfoque teórico dentro del cual nos movemos - el de la escuela socio-histórica, derivada del trabajo de Vygotsky y sus asociados-, sostiene que las habilidades superiores de conocimiento de los sujetos son el resultado de las adquisiciones de conocimientos que se producen en contextos específicos de actividad social. El ordenador es, entonces, concebido como un instrumento educativo más cuya efectividad debe ser juzgada en relación con el uso que de él se haga dentro de las actividades escolares. Ello nos ha llevado a ampliar el ámbito de nuestro trabajo teniendo en cuenta un conjunto de elementos a los cuales nos referiremos más adelante.

Un aspecto determinante en el enfoque de nuestro trabajo es el concepto de "psi- cología práctica", ya avanzado por Vygotsky (1982a), que se refiere tanto a la necesaria relación entre psicología teórica y aplicada, como al valor de verificación que la práctica aplicada ejerce sobre los. instrumentos teóricos. En este sentido nuestra investigación conjuga aspectos teóricos con aspectos aplicados, y ello a través de la generación de instrumentos para la instrucción y del diseño de sistemas de evaluación.

Cuando se trabaja con sujetos con algún tipo de deficiencias existe la tendencia a partir de la consideración de una especial patología que en el plano psicológico se manifiesta en un particular perfil intelectual $-\mathrm{y}$, en ocasiones, de personalidadque tiene consecuencias en los rendimientos escolares. Sin embargo, esta noción recoge un razonamiento tautológico. Por una parte el nivel intelectual se mide a través de pruebas compuestas de tareas muy ligadas a la instrucción escolar, mientras que, por otra, las dificultades que se manifiestan en los aprendizajes escolares tratan de justificarse a través de la existencia de una minusvalía estimada a través de pruebas de rendimiento. Aunque de ninguna manera pretendemos aquí negar la existencia de problemas de aprendizaje y de desarrollo intelectual en sujetos con minusvalías, sí vamos a tratar de ofrecer un enfoque en el que éstas sean consideradas desde otro ángulo.

Nuestra idea es que el estudio de sujetos con deficiencias ofrece la oportunidad de estudiar la incidencia de determinados aspectos del aparato físico del organismo sobre el desarrollo funcional del aparato psíquico. Ello permite avanzar en el estudio de determinados aspectos teóricos de la psicología y, a su vez, fundamenta el desarrollo de técnicas de instrucción y tratamiento que permitan optimizar las posibilidades de desarrollo de los sujetos que sufran esas limitaciones.

Como ya hemos dicho, los sujetos a los que se refiere este trabajo son paralíticos cerebrales. Parafraseando a Robinault y Denhoff (1973) podríamos decir que la parálisis cerebral viene a ser una denominación general para múltiples disfunciones. Se trata, por tanto, de un término clínico genérico que se refiere a la presencia de trastornos fundamentalmente motores de- 
bidos a lesiones en el cerebro inmaduro ya sean pre, peri o post-natales. Estas lesiones pueden afectar -además de a zonas cerebrales responsables de la motricidad, el tono o la coordinación- a las funciones sensoriales, y en ocasiones vienen acompañadas de deficiencia intelectual, de dificultades de aprendizaje, o de alteraciones emocionales o de conducta. No se trata, por consiguiente, de un cuadro específico, sino que bajo esta denominación se recogen trastornos de etiología y gravedad muy dispares.

No se puede, por tanto, hablar de una caracterización peculiar y genérica del desarrollo del paralítico cerebral, sino que es preciso considerar el nivel de aprendizaje de un alumno en relación con la instrucción que ha recibido y recibe y con su nivel de desarrollo psicológico. Todas estas razones nos llevan a considerar a nuestros sujetos -en la línea expresada por Marchesi y Martín (en prensa) - como «alumnos con necesidades educativas especiales" rechazando la idea de "patología del desarrollo" y a sostener una actitud parsimoniosa sobre el efecto de sus lesiones. Es decir, a ser metodológicamente optimistas sobre el efecto de los tratamientos. Desde este punto de vista la función del sistema educativo sería la de atender a esas «necesidades educativas especiales" para que conduzcan al desarrollo más armonioso posible de estos sujetos a través de la organización institucional e instruccional más adecuada.

Una investigación realizada bajo estos supuestos no puede, entonces, únicamente considerar a los alumnos como sujetos, sino que tiene que tener en cuenta múltiples aspectos del entorno, especialmente aquéllos más ligados a los procesos de instrucción como son el modo en que se realiza el trabajo efectivo en el aula y la interacción profesor-alumno durante la instrucción. Esto lo hacemos desde una concepción teórica que sostiene que el desarrollo de las funciones psicológicas y la incorporación de nuevos conocimientos son consecuencia de la realización de actividades en contextos específicos que llegan a provocar la construcción de sistemas funcionales cerebrales (Leontiev, 1959; Luria, 1969). El resultado, al nivel de análisis psicológico, es la aparición de siste- mas complejos de habilidades de interacción que constituirían el equipamiento cognoscitivo del sujeto (Vygotsky, 1982b). El desarrollo, por consiguiente, es una consecuencia del aprendizaje, aunque no es una mera suma de destrezas (Vygostky, 1978).

Este enfoque viene a enfatizar la importancia de los procesos de instrucción sobre la construcción del sujeto humano. Los alumnos con necesidades educativas especiales son sujetos que precisan de unos instrumentos y unos sistemas educativos adaptados a sus necesidades particulares, pero el proceso de aprendizaje y de desarrollo no es radicalmente diferente al de los sujetos denominados "normales», aunque debido a sus handicaps específicos pueda haber algunas desviaciones respecto a la secuencia general de adquisición de destrezas.

\section{Objetivos}

Desde esta perspectiva este trabajo que, insistimos, tiene un carácter exploratorio y fundamentalmente descriptivo, se plantea los siguientes objetivos:

1. Estudiar cuál es el avance de los alumnos en un conjunto de dimensiones relacionadas con el desarrollo cognitivo y los contenidos curriculares de la enseñanza tras un tratamiento escolar de un curso académico completo.

2. Alcanzar una caracterización de algunos aspectos del tratamiento educativo que los profesores desarrollan en el aula.

3. Poner en relación el progreso observado en los conocimientos escolares con algunos aspectos de la intervención educativa que reciben.

Junto a estos aspectos, propios de un estudio sobre los efectos de los procesos de enseñanza-aprendizaje sobre el desarrollo, vamos a explorar la posible influencia que la afectación motriz pudiera tener sobre los rendimientos escolares y el desarrollo. Algunos trabajos han puesto de manifiesto que el nivel de afectación física tiene una importancia limitada sobre el desarrollo cognitivo (Eagle, 1985), y que un adecuado programa de tratamiento en destrezas cognitivas en el que se haga énfasis sobre la utilización de reglas verbales de ac- 
ción permite la adquisición de esas habilidades operatorias incluso cuando la afectación motora es tan importante como para impedir un habla inteligible (Meyers, Coleman y Morris, 1982).

Desde estas consideraciones que se acaban de exponer se plantean las hipótesis y preguntas exploratorias que a continuación se recogen.

\section{Hipótesis}

1. Los sujetos objeto de este estudio presentarán un avance en el dominio de destrezas cognitivas y escolares como resultado de la intervención educativa que han recibido.

2. Estos avances no dependen de un modo lineal del grado de afectación física que sufren.

3. El progreso que pudiere observarse se justificaría por la realización de tareas escolares que exijan un nivel de ejecución superior al que poseían previamente al inicio del tratamiento. En otros términos, tareas de instrucción situadas dentro de la Zona de Desarrollo Próximo (Vygotsky, 1978).

Además de los aspectos recogidos en las hipótesis que acabamos de exponer, nos planteamos el explorar el modo en que se trabaja en el aula y si existen algunos criterios de efectividad de ese trabajo. Por último estudiaremos cuál es el efecto del uso educativo del ordenador relacionándolo con otros aspectos generales de la actividad en el aula.

\section{METODO}

\section{Sujetos}

Catorce niños paralíticos cerebrales en los que no consta la presencia de transtornos sensoriales asociados ni de retrasos intelectuales importantes. Estos niños son alumnos de 3 centros educativos situados en el municipio de Madrid. Uno de ellos específico para este tipo de sujetos y de titularidad privada (sujetos 1 al 5), y los otros dos de titularidad pública, uno con aulas de educación especial para paralíticos cerebrales (sujetos 6 al 10), y el otro acogido al programa de integración del MEC (sujetos 11 al 14).
La tabla 1 recoge las características de los alumnos con los que hemos trabajado.

\section{Procedimiento}

Como ya hemos expresado se trata de un trabajo que trata de evaluar los efectos de un tratamiento educativo sobre el progreso en los conocimientos escolares y el desarrollo intelectual.

Para alcanzar estos objetivos se combinan varias técnicas de trabajo. Por una parte, se sigue un diseño test-retest para evaluar el progreso alcanzado por los alumnos en un curso académico completo (1987-88). Por otra parte, se realizan observaciones de las actividades desarrolladas en el aula, de manera que se muestrea el trabajo que sobre diversas áreas curriculares se lleva a cabo en el curso.

Por lo que se refiere al tratamiento, éste ha sido diseñado por los profesores de cada aula. Para ello han contado con diversos apoyos. Por una parte se les suministró una lista de objetivos curriculares, un protocolo de programación de actividades, así como los resultados obtenidos por cada sujeto en la evaluación realizada a principios de curso. Por otra parte, una vez a la semana un miembro del equipo de investigación tenía una sesión de trabajo con los profesores para seguir la evolución del alumno y comentar el tratamiento.

Hay que señalar que las áreas evaluadas coinciden con la distribución de objetivos curriculares y también vienen recogidas como una de las categorías de codificación en el protocolo de observación.

\section{Material}

Para la evaluación de los sujetos se han utilizado dos instrumentos: el test de las matrices progresivas de Raven adaptado especialmente para su mejor manipulación por parte de los sujetos, y las Pruebas Pedagógicas Graduadas para Preescolar y Ciclo Inicial (PPG) desarrolladas por el Equipo de Asesoramiento Psicopedagógico de Tarrasa que ha sido adaptada para los propósitos de esta investigación.

En todos los casos la evaluación se realizó mediante entrevista individual por profesionales especialista en el trabajo con 
TABLA I

\begin{tabular}{|c|c|c|c|c|c|c|}
\hline \multirow{2}{*}{ Niño } & \multicolumn{2}{|c|}{ Afectación } & \multirow{2}{*}{$\begin{array}{l}\text { Nivel } \\
\text { escolar }\end{array}$} & \multirow{2}{*}{$\begin{array}{c}\text { Historia } \\
\text { escolar }\end{array}$} & \multirow{2}{*}{ Edad } & \multirow{2}{*}{$\begin{array}{c}\text { Inteligencia } \\
\text { (Raven) }\end{array}$} \\
\hline & Motora & Habla & & & & \\
\hline 1 & 2 & 1 & 1 & - & 1 & 2 \\
\hline 2 & 2 & 1 & 1 & 2 & 1 & 3 \\
\hline 3 & 1 & 2 & 3 & 2 & 3 & 3 \\
\hline 4 & 1 & $\overline{1}$ & 2 & - & 3 & 2 \\
\hline 5 & $i$ & 2 & 2 & 2 & 2 & 2 \\
\hline 6 & 2 & 2 & 3 & - & 3 & - \\
\hline 7 & 2 & 1 & 2 & 1 & 3 & 2 \\
\hline 8 & 2 & 2 & 2 & 3 & 2 & 1 \\
\hline 9 & 2 & 1 & 1 & 1 & 1 & 1 \\
\hline 10 & 3 & 3 & 1 & 1 & 1 & 1 \\
\hline 11 & 3 & 3 & 1 & 3 & 1 & 3 \\
\hline 12 & 3 & 3 & 2 & 3 & 2 & 1 \\
\hline 13 & 3 & 3 & 3 & 3 & 2 & 3 \\
\hline 14 & 3 & 3 & 1 & 3 & 1 & 2 \\
\hline
\end{tabular}

Los criterios para asignar las puntuaciones recogidas en esta tabla ban sido los siguientes:

Afectación motora: se refiere al grado de motricidad funcional en los miembros superiores. 3 indica capacidad de manipulación fina; 2 indica capacidad de manipulación gruesa, $y 1$ indica tan sólo la capacidad de señalar.

Afectación del habla: indica el grado de inteligibilidad de su babla. 3 indica babla comprensible; 2 babla dificilmente comprensible; 1 no dispone de babla inteligible.

Nivel escolar: 1 corresponde a preescolar; 2 a primero de EGB y 3 a segundo y tercero de $E G B$.

Historia escolar: trata de recoger la relación entre el nivel escolar actual y el número de años de escolarización previa. Es decir, una estimación del aprovechamiento escolar. Para ello se ha ideado una proporción en la que el numerador lo constituye el nivel escolar actual (considerando a primero de preescolar como nivel 1, primero de EGB como nivel 3, etc.), y como denominador el número de años que ba asistido a la escuela en estos niveles educativos. De este modo se ha construido una escala ordinal cuyas puntuaciones corresponden a los intervalos de proporción que a continuación se indican. La puntuación $* 3$ " indica una proporción igual a 1 (p. e., 4/4,3/3, 1/1, etc.), la puntuación *2* indica una proporción superior a 0,60 e inferior a 0,83 (p. e., 2/3, 5/6,3/5); la puntuación * $1 *$ una proporción superior a 0,43 e inferior a 0,50 (p. e., 1/2,3/7).

Edad: El nivel 1 lo constituyen sujetos de 5 y 6 años; el nivel 2, entre 7 y 9 años y el nivel 3 los niños de 10 y 11 años.

Inteligencia: Se han establecido tres grupos atendiendo a sus puntuaciones directas en el test de Raven aplicado a principios del curso académico objeto de la investigación. Cada uno de los grupos representa puntuaciones obtenidas en los intervalos que a continuación se relacionan: $1=3-9 ; 2=10-15 ; 3=16-20$. La razón de utilizar las puntuaciones directas y no las baremadas está en las dificultades de aplicar los baremos normalizados a las poblaciones con problemas especiales.

este tipo de sujetos, garantizándose la comunicación con éstos mediante el uso de las técnicas adecuadas (señalamiento, rastreo verbal, etc.).

La observación en el aula fue realizada por un observador externo y recogida en forma narrativa y luego transcrita al protocolo recogido en la Figura 1 de donde posteriormente se coficaba.
Un ejemplar del mismo protocolo era utilizado por el profesor para programar la actividad a realizar en el período que iba a ser observado 4 .

Cada sujeto fue observado con una frecuencia aproximada de una hora a la semana, tratándose de cubrir todas las áreas objeto de los contenidos curriculares e indicándosele a los profesores que los perío- 
dos de observación deberían de ser representativos de la actividad cotidiana en el aula. Hay que señalar que el $100 \%$ de las tareas observadas recogían interacciones diádicas profesor-alumno o entre compañeros.

Se realizaron un total de 241 horas de observación para el total de los sujetos. La unidad de análisis utilizada fue la tarea escolar correspondiente a un objetivo instruccional. El número total de tareas analizadas fue de 358 .

\section{RESULTADOS}

Por el tipo de procedimiento seguido durante nuestro trabajo, vamos a presentar los resultados obtenidos en función de una secuencia en la que se deja traslucir el sentido de nuestro análisis de los mismos.

Así vamos a empezar exponiendo los resultados relativos a la ganancia en habilidades y conocimientos curriculares obtenida por los sujetos del estudio tras un curso académico. A continuación analizaremos la relación existente entre dicha ganancia y una serie de variables relevantes tales como el grado de afectación motriz, la calidad de su habla, la edad, el nivel escolar, etc.

Tras este primer bloque de resultados, se presentan los relativos al estudio del trabajo realizado dentro del aula del que se ha extraído una muestra de observaciones. Finalmente, se exponen los resultados de los análisis que tratan de poner en relación los dos bloques anteriores, es decir, los resultados relativos a la ganancia experimentada por los sujetos tras un curso académico y aquéllos que se refieren al estudio del trabajo diario dentro del aula recogidos mediante observación.

\section{Ganancia tras un curso académico}

Para el análisis del progreso realizado por los sujetos de la muestra tras un curso escolar, se tomaron dos medidas - al principio y al final del curso- mediante dos instrumentos de evaluación diferentes. El primero fue el test de matrices progresivas de Raven (Escala color) -en una versión experimental adaptada a las características de la capacidad manipulativa de los sujetos de la muestra- y el segundo la escala
«Pruebas Pedagógicas Graduadas para preescolar y ciclo inicial» (PPG), asimismo adaptada para su utilización con deficientes motóricos severos.

En la Tabla 2 se presentan las puntuaciones obtenidas por los catorce sujetos de la muestra, al principio y al final del curso, en cada una de las pruebas mencionadas, aunque en el caso de las PPG se muestran las puntuaciones obtenidas en ocho aspectos parciales además de una puntuación global. También aparecen las medias de las diferencias antes-después así como las desviaciones típicas de tales diferencias.

Las puntuaciones de las PPG tratan de expresar, para los cuatro cursos en los que son aplicables estas pruebas, la suma de la proporción de elementos de la escala que el niño soluciona correctamente dentro de cada uno de los cuatro cursos. De este modo, una puntuación entre cero y uno expresa que el sujeto se encuentra, dentro del aspecto que la escala evalúe, en un nivel de primero de preescolar. Si puntua entre uno y dos implica un nivel de segundo de preescolar. Si entre dos y tres, primero de ciclo inicial y si lo hace entre tres y cuatro, segundo de ciclo inicial.

Sabiendo esto, se puede apreciar en la mencionada tabla que los niveles de desarrollo de los niños dentro de los diferentes aspectos evaluados suelen ser muy irregulares, destacando, sobre todo, el bajo nivel de desarrollo de la capacidad de expresión verbal y la ausencia de relación de ésta con los niveles de desarrollo alcanzados en comprensión y lecto-escritura. En cualquier caso va a ser el análisis de las diferencias medias obtenidas por todo el grupo en la comparación antes-después el que nos permita profundizar más en el significado de esta tabla.

La medida de la ganancia se ha realizado mediante la resta entre las puntuaciones obtenidas al principio y al final del curso. La cuantía media de tales diferencias, y su significación, en cada uno de los aspectos que miden las «Pruebas Psicopedagógicas Graduadas", en la suma de todos ellos en una única escala y en el Raven, se presenta en la Tabla 3.

Dicha tabla pone de manifiesto que, tomados en su conjunto, los sujetos de nuestra muestra mejoraron significativamente tras un curso escolar en lo que se refiere a 
TABla II

Puntuaciones obtenidas por cada uno de los sujetos de la muestra en las diferentes pruebas aplicadas en el diseño antes-después

\begin{tabular}{|c|c|c|c|c|c|c|c|c|}
\hline \multirow{2}{*}{ Sujeto } & \multicolumn{2}{|c|}{ Raven } & \multicolumn{2}{|c|}{ Perc. visual } & \multicolumn{2}{|c|}{ Perc. auditiva } & \multicolumn{2}{|c|}{ Esq. corporal } \\
\hline & Pre. & Post. & Pre. & Post. & Pre. & Post. & Pre. & Post. \\
\hline 1 & 11 & 11 & 0,200 & 0,800 & 0,000 & 0,000 & 0,000 & 0,000 \\
\hline 2 & 20 & 28 & 2,000 & 2,666 & 1,400 & 1,800 & 1,500 & 2,166 \\
\hline 3 & 17 & 20 & 3,500 & 4,000 & 2,750 & 2,750 & 3,570 & 3,570 \\
\hline 4 & 10 & 12 & 1,000 & 2,000 & 1,600 & 2,250 & 2,666 & 3,142 \\
\hline 5 & 15 & 12 & 1,600 & 1,600 & 1,400 & 2,000 & 2,666 & 3,280 \\
\hline 6 & 14 & 13 & 2,000 & 2,666 & 1,400 & 1,800 & 2,000 & 2,000 \\
\hline 7 & - & 16 & 3,500 & 4,000 & 1,600 & 2,250 & 3,280 & 3,140 \\
\hline 8 & 9 & 11 & 2,000 & 3,000 & 1,600 & 1,600 & 2,333 & 2,830 \\
\hline 9 & 3 & 9 & 0,000 & 0,000 & 0,000 & 0,333 & 0,100 & 0,300 \\
\hline 10 & 8 & 11 & 0,600 & 1,200 & 1,000 & 1,250 & 0,900 & 1,666 \\
\hline 11 & 16 & 22 & 1,600 & 2,000 & 1,600 & 2,250 & 2,333 & 3,000 \\
\hline 12 & 6 & 7 & 1,000 & 1,400 & 1,200 & 1,400 & 1,666 & 2,000 \\
\hline 13 & 17 & 18 & 3,500 & 4,000 & 3,000 & 3,000 & 3,142 & 3,428 \\
\hline 14 & 13 & 14 & 2,000 & 2,666 & 1,000 & 1,200 & 1,500 & 2,000 \\
\hline \multirow{2}{*}{\multicolumn{2}{|c|}{$\begin{array}{l}\text { Media de la dif. } \\
\mathrm{S}_{\mathrm{s}}\end{array}$}} & 2,23 & \multirow{2}{*}{\multicolumn{2}{|c|}{$\begin{array}{l}0,534 \\
0,280\end{array}$}} & \multirow{2}{*}{\multicolumn{2}{|c|}{$\begin{array}{l}0,305 \\
0,248\end{array}$}} & \multicolumn{2}{|c|}{0,348} \\
\hline & & 3,03 & & & & & & \\
\hline
\end{tabular}

\begin{tabular}{|c|c|c|c|c|c|c|}
\hline \multirow{2}{*}{ Sujeto } & \multicolumn{2}{|c|}{ Lógica matem. } & \multicolumn{2}{|c|}{ Cálculo y Gr. de Nos. } & \multicolumn{2}{|c|}{ Comprensión Verbal } \\
\hline & Pre. & Post. & Pre. & Post. & Pre. & Post. \\
\hline 1 & 0,000 & 0,166 & 0,000 & 0,333 & 0,250 & 0,250 \\
\hline 2 & 1,600 & 2,000 & 1,570 & 2,125 & 1,750 & 1,750 \\
\hline 3 & 3,750 & 4,000 & 3,700 & 4,000 & 4,000 & 4,000 \\
\hline 4 & 1,600 & 2,000 & 1,570 & 2,000 & 2,250 & 3,333 \\
\hline 5 & 1,800 & 1,800 & 1,570 & 1,570 & 2,750 & 3,333 \\
\hline 6 & 3,000 & 1,800 & 1,420 & 2,125 & 2,500 & 3,333 \\
\hline 7 & 3,250 & 3,500 & 2,125 & 2,875 & 4,000 & 4,000 \\
\hline 8 & 1,000 & 2,000 & 1,420 & 2,125 & 1,750 & 2,750 \\
\hline 9 & 0,160 & 0,160 & 0,333 & 0,333 & 1,000 & 1,250 \\
\hline 10 & 0,500 & 1,000 & 0,000 & 1,280 & 0,500 & 1,000 \\
\hline 11 & 1,600 & 2,000 & 1,850 & 2,000 & 1,750 & 2,000 \\
\hline 12 & 0,666 & 0,830 & 1,850 & 1,850 & 1,000 & 0,750 \\
\hline 13 & 1,800 & 2,000 & 2,750 & 3,100 & 2,500 & 4,000 \\
\hline 14 & 1,400 & 1,400 & 1,142 & 1,000 & 1,000 & 2,000 \\
\hline \multicolumn{2}{|c|}{$\begin{array}{l}\text { Media de la dif. } \\
\mathrm{S}_{\mathrm{x}}\end{array}$} & $\begin{array}{l}0,182 \\
0,457\end{array}$ & \multicolumn{2}{|c|}{$\begin{array}{l}0,386 \\
0,372\end{array}$} & \multicolumn{2}{|c|}{$\begin{array}{l}0,479 \\
0,500\end{array}$} \\
\hline
\end{tabular}

la medida del Raven y a la medida total de la PPG. Cuando esta última escala se analiza teniendo en cuenta cada uno de los ocho aspectos de que se compone, se observa que en todos ellos existe una ganancia significativa excepto en el de lógicamatemática.

En la discusión volveremos sobre este hecho.

\section{Influencia de variables del sujeto}

El siguiente paso en el proceso de análisis lo constituye el estudio de la influencia que determinado tipo de variables de los sujetos pudieran tener sobre la cuantía de la ganancia total lograda. Concretamente, se ha estudiado la influencia del nivel de afectación motriz de los sujetos, su 
TABLA II

(Cont.)

\begin{tabular}{|c|c|c|c|c|c|c|}
\hline \multirow{2}{*}{ Sujeto } & \multicolumn{2}{|c|}{ Expresión verbal } & \multicolumn{2}{|c|}{ Lecto-escritura } & \multicolumn{2}{|c|}{ PPG Total } \\
\hline & Pre. & Post. & Pre. & Post. & Pre. & Post. \\
\hline 1 & 0,000 & 0,000 & 0,000 & 0,000 & 0,055 & 0,194 \\
\hline 2 & 0,000 & 0,000 & 1,000 & 1,500 & 1,512 & 1,923 \\
\hline 3 & 3,333 & 3,666 & 2,500 & 2,375 & 3,400 & 3,540 \\
\hline 4 & 0,000 & 0,250 & 1,250 & 1,750 & 1,560 & 2,160 \\
\hline 5 & 0,500 & 0,750 & 1,000 & 1,000 & 1,710 & 1,970 \\
\hline 6 & 0,000 & 0,000 & 1,250 & 1,500 & 1,690 & 1,920 \\
\hline 7 & 0,000 & 0,000 & 1,750 & 1,750 & 2,320 & 2,590 \\
\hline 8 & 0,000 & 0,000 & 1,000 & 1,500 & 1,530 & 2,027 \\
\hline 9 & 0,000 & 0,000 & 0,000 & 0,000 & 0,194 & 0,300 \\
\hline 10 & 0,250 & 0,500 & 0,000 & 0,000 & 0,580 & 1,128 \\
\hline 11 & 0,750 & 0,750 & 1,000 & 1,250 & 1,640 & 2,000 \\
\hline 12 & 0,250 & 0,750 & 1,000 & 1,000 & 1,153 & 1,333 \\
\hline 13 & 3,666 & 3,666 & 3,500 & 3,750 & 3,000 & 3,370 \\
\hline 14 & 0,000 & 2,000 & 0,000 & 1,000 & 1,153 & 1,590 \\
\hline \multirow{2}{*}{\multicolumn{2}{|c|}{$\begin{array}{l}\text { Media de la dif. } \\
\mathrm{S}_{\mathrm{x}}\end{array}$}} & & \multirow{2}{*}{\multicolumn{2}{|c|}{$\begin{array}{l}0,223 \\
0,299\end{array}$}} & \multirow{2}{*}{\multicolumn{2}{|c|}{$\begin{array}{l}0,333 \\
0,157\end{array}$}} \\
\hline & & & & & & \\
\hline
\end{tabular}

capacidad de habla, su edad, el nivel escolar en el que se encuentran actualmente y, derivado de éste, su nivel de aprovechamiento escolar estimado mediante la razón entre los años de escolarización y el nivel alcanzado. Finalmente, se estudia también la posible influencia de las capacidades intelectuales de los sujetos estimadas mediante su puntuación en el test de matrices progresivas de Raven. En definitiva, se utilizan como variables independientes en este análisis las relativas al estado de los sujetos de la muestra tal y como aparecen en la Tabla 1.
Para llevar a cabo este estudio se ha tomado como variable dependiente la diferencia, para cada sujeto, entre su puntuación total en las PPG al principio y al final del curso. En función de su nivel en cada una de las seis variables independientes mencionadas se divide la muestra en tres grupos distintos para cada análisis. La prueba estadística utilizada ha sido la de Kruskal-Wallis, alternativa no paramétrica al análisis de varianza de un factor de efectos fijos completamente aleatorizado. Mediante dicha prueba se puede comprobar si existen diferencias entre los tres gru-

TABLA III

Significación de las diferencias obtenidas tras un curso académico para cada una de las medidas realizadas

\begin{tabular}{lccccc}
\hline & Raven & $\begin{array}{c}\text { PPG } \\
\text { total }\end{array}$ & $\begin{array}{c}\text { Percepción } \\
\text { visual }\end{array}$ & $\begin{array}{c}\text { Percepción } \\
\text { auditiva }\end{array}$ & $\begin{array}{c}\text { Esquema } \\
\text { corporal }\end{array}$ \\
\hline $\begin{array}{l}\text { Dif. media } \\
\text { Probabilidad }\end{array}$ & $\begin{array}{c}2,23 \\
<0,05\end{array}$ & $\begin{array}{c}0,333 \\
<0,01\end{array}$ & $\begin{array}{c}0,534 \\
<0,01\end{array}$ & $\begin{array}{c}0,305 \\
<0,01\end{array}$ & $\begin{array}{c}0,348 \\
<0,01\end{array}$ \\
\hline & $\begin{array}{c}\text { Lógica } \\
\text { matemática }\end{array}$ & $\begin{array}{c}\text { Cálculo } \\
\text { y grafia }\end{array}$ & $\begin{array}{c}\text { Comprensión } \\
\text { verbal }\end{array}$ & $\begin{array}{c}\text { Expresión } \\
\text { verbal }\end{array}$ & $\begin{array}{c}\text { Lecto- } \\
\text { escritura }\end{array}$ \\
\hline $\begin{array}{l}\text { Dif. media } \\
\text { Probabilidad }\end{array}$ & $\begin{array}{c}0,182 \\
<0,10\end{array}$ & $\begin{array}{c}0,386 \\
<0,01\end{array}$ & $\begin{array}{c}0,479 \\
<0,01\end{array}$ & $\begin{array}{c}0,255 \\
<0,05\end{array}$ & $\begin{array}{c}0,223 \\
<0,01\end{array}$ \\
\hline
\end{tabular}


TABLA IV

Influencia de las variables del sujeto sobre el nivel de ganancia alcanzado tras un curso escolar

(Estadistica de Kruskal-Wallis y probabilidad asociada al mismo)

\begin{tabular}{lclllll}
\hline & $\begin{array}{c}\text { Afectación } \\
\text { motriz }\end{array}$ & Habla & Edad & $\begin{array}{c}\text { Nivel } \\
\text { escolar }\end{array}$ & $\begin{array}{l}\text { Aprov. } \\
\text { escolar }\end{array}$ & Raven \\
\hline $\mathrm{H}$ & 2,19 & 1,08 & 1,84 & 1,21 & 1,22 & 0,04 \\
Prob. & 0,333 & 0,582 & 0,397 & 0,546 & 0,543 & 0,977 \\
\hline
\end{tabular}

pos obtenidos según cada una de las seis variables, de tal modo que la existencia de tales diferencias implicaría una influencia significativa de la variable mediante la que se construyen los grupos sobre la dependiente, en este caso, sobre el nivel de ganancia logrado. En la Tabla 4 se presentan los resultados obtenidos (valor del estadístico y su probabilidad de aparición bajo la hipótesis de que no existen diferencias entre los tres grupos) para cada una de las seis variables mencionadas anteriormente.

Los resultados ponen de manifiesto que ninguna de las seis variables tomadas en consideración influye significativamente sobre el nivel de ganancia alcanzado (las probabilidades son todas mayores que $0,05)$, si bien parece que no todas arrojan los mismos resultados. Asi puede apreciarse que el nivel intelectual es la variable que menores diferencias produce entre los tres grupos, mientras que la edad y el nivel de afectación serían las que más, si bien todavia la cuantia de tales diferencias está muy alejada del nivel de significación.

\section{Análisis del proceso de instrucción en el aula}

Para poder establecer en qué medida la ganancia observada tras el paso de un curso académico está relacionada con el tipo de trabajo que se realiza dentro del aula, se llevó a cabo una observación en dicho contexto para tratar de determinar cuáles son las variables relevantes del proceso de instrucción que explican el rendimiento de los sujetos en sus tareas escolares y, por tanto $y$ en la medida en que la observación realizada sea una muestra representativa de todo el trabajo realizado durante el curso, el nivel de la ganancia obtenida.
Junto con ello, y en relación con nuestro interés por las posibilidades que su uso pudiera tener en este contexto, se ha estudiado también el modo en el que las maestras utilizan el ordenador en su trabajo dentro del aula y la posible influencia de dicho uso sobre el rendimiento en las tareas escolares.

Por lo que a la primera cuestión planteada se refiere se ha tratado de correlacionar todas las variables observadas con el nivel de ejecución de la tarea por parte del niño, que sería la variable dependiente a explicar en este caso.

Hay que señalar que dentro del tipo de variables analizadas se pueden distinguir dos tipos: aquéllas que pueden ser medidas en un nivel de intervalo y aquéllas que sólo pueden ser categorizadas nominalmente. Dentro del primer grupo se encuentran las variables relativas a los tipos de mensajes motivacionales (tipo de atribuciones y valoraciones) que la maestra da al niño durante el proceso de ejecución de la tarea, el sistema de incentivos utilizado y el tipo de ayudas (preguntas o instrucciones) dadas al niño en el proceso de realización de su tarea. Dentro del segundo grupo se consideran las siguientes variables: adecuación del análisis que realiza el niño de la tarea (buena o mala), sistema semiótico utilizado de forma predominante (acción, dibujo, símbolo, habla), modo de comunicación (habla, habla asistida, escritura, escritura asistida, sistema de comunicación no vocal, código personal de comunicación), grado de dificultad de la tarea para el niño (por debajo de sus posibilidades, dentro de su nivel actual, algo por encima de su nivel, muy por encima de su nivel), ajuste entre el objetivo que se pretende trabajar y la tarea que se propone para ello (ajuste, no ajuste), adecuación 
de la consigna dada por la maestra a las características de la tarea (se adecúa, no se adecúa), cantidad de ayudas dadas por la maestra en relación al tipo de tarea (no ayuda, ayuda en un paso del proceso de realización, ayuda en varios, ayuda en todos). La calidad de la ejecución de la tarea por parte del niño se evalúa también mediante un sistema de tres categorías: buena, regular, mala.

Los resultados muestran que ninguna de las variables consideradas dentro del primero de estos grupos correlaciona significativamente con el nivel de ejecución de la tarea por lo que obviamos su presentación, aunque más tarde volveremos sobre este hecho. Para el segundo grupo de variables (las medidas a un nivel nominal), en la Tabla 5 se presenta la cuantía de sus correlaciones con el nivel de ejecución, así como la significación de tales correlaciones.

En esta tabla se observa como las variables que correlacionan significativamente con el resultado obtenido en la ejecución de la tarea son la adecuación del análisis que hace el niño de la tarea (a mejor análisis mejor ejecución), el sistema semiótico utilizado (la ejecución es mejor cuando se utiliza un sistema de símbolos que en los otros tres casos, y mejor cuando se utiliza el habla o el dibujo que cuando se trabaja sólo mediante la acción), el grado de dificultad (a mayor dificultad peor ejecución) y la adecuación de la tarea al objetivo propuesto (a mejor adecuación mejor ejecución).

Sin embargo, un hecho que puede quedar enmascarado por estos datos es el de la posible influencia indirecta de unas va- riables a través de otras. Un modo de ver este hecho es analizar las correlaciones existentes entre estas cuatro variables que correlacionan significativamente con la ejecución. Además hay que tener en cuenta que la distribución de las frecuencias en las variables no es homogénea. Así, el $92,4 \%$ de las veces el análisis de la tarea realizado por el niño es adecuado y el $95,5 \%$ de las veces la tarea se adecúa a su objetivo, de lo que se desprende que aún correlacionando significativamente con el nivel de ejecución dichas variables no nos permiten explicar las diferencias en la ejecución existente en más del $90 \%$ de los casos.

Si no consideramos, entonces, estas dos variables, quedarían las otras dos como responsables del nivel de ejecución. Volviendo sobre lo que comentábamos anteriormente, el análisis de la correlación entre estas dos variables $(C=0,241$; $\mathrm{p}<0,01)$ pone de manifiesto que el trabajo con aquellos sistemas que beneficiaban la ejecución (símbolos) se realiza la mayor parte de las veces dentro de los grados de dificultad más bajos y, por el contrario, el trabajo mediante sistemas que arrojan un menor nivel de ejecución se hace dentro del grado de dificultad más alto en la mayor parte de los casos.

De todo ello se desprendería que de entre todas las variables consideradas dentro del proceso de ejecución de una tarea en el aula, es el nivel de dificultad de la misma el que mejor explica el resultado.

Ahora bien, si partimos del concepto de zona de desarrollo próximo podemos considerar que el trabajo adecuado para el

TABLA V

Coeficiente de contingencia entre las variables medidas a nivel nominal y el nivel de ejecución en la tarea

\begin{tabular}{lccccc}
\hline & $\begin{array}{c}\text { Adecuación } \\
\text { análisis }\end{array}$ & $\begin{array}{c}\text { Sistema } \\
\text { semiótico }\end{array}$ & $\begin{array}{c}\text { Modo de } \\
\text { comunicación }\end{array}$ & $\begin{array}{c}\text { Grado de } \\
\text { dificultad }\end{array}$ & $\begin{array}{c}\text { Adecuación } \\
\text { objetivo/tarea }\end{array}$ \\
\hline C & 0,247 & 0,201 & 0,165 & 0,265 & 0,151 \\
Probabilidad & $<0,0001$ & 0,0207 & 0,2662 & 0,0002 & 0,0155 \\
\hline & $\begin{array}{c}\text { Adecuación } \\
\text { consigna/tarea }\end{array}$ & Ayudas & & & \\
\hline C & 0,080 & 0,164 & & \\
Probabilidad & 0,3225 & 0,1329 & & \\
\hline
\end{tabular}


prögreso del niño es aquél que se realiza un poco por encima de su nivel actual de posibilidades. Teniendo en cuenta este hecho hemos llevado a cabo un análisis posterior en el que se han eliminado todas las secuencias de observación relativas a las tareas realizadas muy por encima de las posiblidades del sujeto o por debajo de las mismas. Hemos agrupado las secuencias restantes según estuvieran dentro de su nivel actual o algo por encima tratando de comparar lo que ocurre cuando se trabaja dentro de la zona y cuando no. Esta comparación se ha realizado tomando como variables dependientes la ejecución y aquellas variables del grupo de las medidas a nivel de intervalo y que no habían mostrado relación con el mencionado nivel de ejecución.

Las diferencias entre los dos grupos de observaciones se presentan en la Tabla 6 . En el caso del nivel de ejecución hemos utilizado un estadístico no paramétrico (recuérdese que esta variable se evaluaba mediante una escala nominal compuesta por tres categorías - mal, regular, bien-), mientras que para el resto de las variables hemos realizado un contraste sobre diferencia de medias para muestras independientes.

Los resultados de dicha comparación ponen de manifiesto que no existen diferencias entre los dos grupos de secuencias de observación por lo que se refiere al nivel de ejecución alcanzado $\left(\mathrm{X}^{2}=0,278\right.$; $\mathrm{p}>0,50$ ). Sí existen diferencias, sin embargo, a favor de los que trabajan dentro de la zona de desarrollo próximo en cuanto que reciben mejores mensajes motivacionales: más comparaciones consigo mismo $\left(t_{3,170}=-2,24 ; p<0,05\right)$, más atribuciones internas $\left(t_{9,138}=-3,21 ; p<0,005\right)$ y más atribuciones controlables $\left(t_{3.158}=-2,09 ; p<0,05\right)$; se utilizan mejores sistemas de incentivos; más refuerzos sociales $\left(t_{5,95}=-2,5 ; p<0,05\right)$, aunque también reciben más esfuerzos verbales negativos $\left(t_{1,109}=-2,93 ; p<0,005\right) y$ reciben más ayudas durante el proceso: más instrucciones y preguntas durante la ejecución $\left(t_{1,140}=-2,39 ; \mathrm{p}<0,05\right.$ y $t_{8,116}=-2,6 ; p<0,05$ respectivamente) $y$

\section{TABLA VI}

Diferencias en el estilo instruccional espontáneo del profesor en tareas con dos niveles de dificultad: asimilables a la Zona de Desarrollo Actual (ZDA) y a la Zona de Desarrollo Próximo (ZDP)

\begin{tabular}{ccccc}
\hline Variable & media ZDA & media ZDP & Diferencia & P. \\
\hline $\begin{array}{l}\text { Rendimiento en las tareas } \\
\begin{array}{l}\text { Contraste entre tareas con alto } \\
\text { bajo rendimiento }\end{array}\end{array}$ & 1,523 & 1,406 & 0,117 & 0,1359 \\
$\mathrm{X}^{2}=3,841$ & $\mathrm{X}^{2}=0,278$ & $\mathrm{p}>0,50$ & $=0,05$
\end{tabular}

Mensajes motivacionales

Comparaciones con uno mismo

Atribuciones internas

$\begin{array}{llll}0,1765 & 0,3579 & 0,1814 & 0,0267 \\ 0,1412 & 0,4316 & 0,2904 & 0,0017 \\ 0,1588 & 0,3263 & 0,1675 & 0,0383\end{array}$

Atribuciones controlables

0,1588

0,3263

0,1675

0,0383

\section{Sistema de incentivos}

Respuestas positivas del niño al refuerzo social

$\begin{array}{llll}0,0414 & 0,2967 & 0,2553 & 0,0142 \\ 0,1294 & 0,5208 & 0,3914 & 0,0042\end{array}$

Refuerzos verbales negativos

\section{Ayudas en la instrucción}

Instrucciones durante la ejecución

Preguntas durante la ejecución

Conductas de modelado 
más mensajes de modelado $\left(\mathrm{t}_{7,165}=-2,11 ; \mathrm{p}<0,05\right)$. Sobre todo ello volveremos en la discusión.

Por último, y por lo que al análisis del uso que del ordenador se hace dentro del aula, vamos a comentarlo en relación con las dos variables más relevantes según lo visto hasta ahora: el grado de dificultad de la tarea y el nivel de ejecución del niño en la misma.

El primer dato a destacar es el porcentaje de uso que se hace de él, tomando como punto de referencia la totalidad de tareas registradas durante la observación. El ordenador, según esto, se ha usado el $16,5 \%$ de las tareas que se han registrado. Teniendo en cuenta este porcentaje, en la Tabla 7 se presenta la comparación, en cuanto a las dos variables mencionadas, con el resto de tareas que se hacen sin ordenador.

En dicha tabla se puede observar que no existen diferencias apreciables en cuanto al nivel de ejecución entre aquellas tareas que se realizan mediante ordenador y las que se realizan sin él. Sin embargo, se aprecia claramente que el reparto de las tareas según el nivel de dificultad es distinto cuando se usa el ordenador que cuando no se usa. Dadas las relaciones que aparecían en los análisis anteriores esto implicaría que dentro de los casos en que se usa el ordenador las relaciones entre dificultad y ejecución podrían darse de manera distinta que en el resto de los casos.
Resultados en cuanto a las relaciones existentes entre el trabajo en el aula y la ganancia obtenida tras un curso escolar

En el último paso de nuestros análisis se ha tratado de estudiar, al igual que lo hacíamos anteriörmente con respecto a aquellas variables que hemos denominado «del sujeto», las relaciones existentes entre las variables intervinentes dentro de la dinámica de trabajo en el aula y la ganancia mostrada por los sujetos de la muestra.

Dado que parece claro que la calidad en la realización de la tarea por parte del niño depende fundamentalmenta del nivel de dificultad de la misma y dado que, como hemos visto, el trabajo dentro de lo que podríamos denominar zona de desarrollo próximo, según hemos operativizado nuestros datos, parecía producir un estilo más adecuado de instrucción en cuanto a mensajes motivacionales, sistemas de incentivos y tipo de ayudas, hemos considerado que un modo de poner en relación la dinámica de trabajo en el aula y la cuantía de la ganancia obtenida tras un curso académico era el utilizar como variable independiente el porcentaje de tareas que se trabajaba con cada niño dentro de la zona de desarrollo próximo. Asimismo, hemos considerado la posibilidad de que tal ganancia estuviera relacionada con las diferencias entre el modo de trabajar de las maestras o de los centros escolares.

Nuevamente, la técnica utilizada para

\section{TABLA VII}

Comparación en cuanto a grado de dificultad y nivel de ejecución entre los casos en los que se utiliza el ordenador y aquéllos en los que no se utiliza

Grado de dificultad

\begin{tabular}{lcccc}
\cline { 2 - 4 } & Por debajo & Nivel actual & Algo encima & $\begin{array}{c}\text { Muy por } \\
\text { encima }\end{array}$ \\
\hline Sin ordenador & $18,7 \%$ & $52,7 \%$ & $25,5 \%$ & $3,1 \%$ \\
Con ordenador & $36,2 \%$ & $25,9 \%$ & $36,2 \%$ & $1,7 \%$ \\
\hline \multicolumn{5}{c}{ Nivel de ejecución } \\
\cline { 2 - 5 } & Malo & Regular & Bueno \\
\hline Sin ordenador & $7,0 \%$ & \multicolumn{2}{c}{$34,9 \%$} & $58,1 \%$ \\
Con ordenador & $6,9 \%$ & \multicolumn{2}{c}{$41,4 \%$} & $51,7 \%$ \\
\hline
\end{tabular}


TABLA VIII

Relaciones entre las variables relativas al proceso de instrucción y la cuantia de la ganancia obtenida

\begin{tabular}{lccc}
\hline & $\begin{array}{c}\text { \% de trabajo } \\
\text { zona de d.p. }\end{array}$ & Maestra & Centro escolar \\
\hline H & 3,93 & 3,32 & 0,86 \\
Probabilidad & 0,139 & 0,853 & 0,648 \\
\hline
\end{tabular}

este análisis ha sido la prueba no paramétrica de Kruskal-Wallis. La muestra se ha dividido en tres grupos para el caso del estudio de las variables de porcentaje de trabajo en la zona de desarrollo próximo y tipo de centro. Para el caso de la variable maestra se han hecho tantos grupos como maestras había. En la Tabla 8 se muestran los resultados obtenidos al respecto.

Dichos resultados muestran que ninguna de las tres variables consideradas arroja diferencias significativas en cuanto al nivel de ganancia aún cuando el porcentaje de trabajo dentro de la zona de desarrollo próximo se acerca bastante al nivel de significación. Comparativamente con el resto de ellas consideradas, se puede decir que esta variable es la que más se acerca a la posibilidad de explicar la ganancia experimentada por los sujetos. En cualquier caso, se hace necesaria una visión global de todo el estudio para tratar de sacar el mayor partido posible a los resultados hasta ahora expuestos. Con ello entramos ya en la discusión de los mismos.

\section{DISCUSION}

Los resultados que acabamos de exponer están de acuerdo con lo expuesto en la primera de las hipótesis arriba recogidas. Se observa un aumento general del rendimiento en las pruebas criteriales de conocimientos escolares y de desarrollo cognitivo. Sin embargo, dadas las características peculiares de los sujetos con los que hemos trabajado conviene discutir la cuantía de estos avances.

El progreso medio alcanzado es equivalente, en términos criteriales, a un tercio de curso académico, algo a todas luces insuficiente pero que debe ser matizado en relación con el tipo de tratamiento administrado y que comentaremos más adelan- te. En todas las escalas se aprecia también un aumento significativo de rendimiento, con excepción de la correspondiente a lógica matemática, algo que puede ser explicable por el artefacto de medida, toda vez que en este caso un solo ítem cuenta como todo un curso académico por lo que la capacidad de discriminación en esta escala en concreto es pequeña. Los aspectos relacionados con la producción de lenguaje (expresión verbal y lecto-escritura) son los que presentan un progreso más lento, algo que no resulta sorprendente si tenemos en cuenta las limitaciones motoras de los sujetos. Los que ya resulta más difícil de explicar es el por qué de la falta de progreso en lectura, pero sobre ello volveremos también más adelante cuando nos refiramos al progreso en habilidades como consecuencia de la instrucción.

Si nos fijamos en las puntuaciones finales de los sujetos y las ponemos en relación con la edad podemos darnos cuenta de que el nivel de los sujetos está muy por debajo de lo esperable para su edad; si tomamos como referente el criterio de la población normal hay un retraso medio de alrededor de tres años. Sin embargo, la variabilidad es muy grande, pues cinco sujetos presentan un retraso de sólo un año, o no tienen ninguno en absoluto, mientras que por otro lado otros cinco sujetos tienen retrasos que oscilan entre los cuatro o cinco años.

Estos datos que acabamos de comentar deben ponerse en relación con la edad de inicio de la escolarización, tan sólo dos sujetos han ingresado en la escuela a edades muy superiores a la criterial, en concreto con un retraso de tres años, estando ambos dentro del grupo de los que presentan un mayor retraso en nivel de rendimiento, si bien hay que matizar que el progreso observado en estos dos sujetos está en- 
tre los más altos de todo el grupo estudiado.

En cualquier caso hay que hacer notar que existe un progreso significativo en los conocimientos de los sujetos, aunque éste esté por debajo del criterio, sin que dispongamos de datos que permitan comparar el aumento de rendimiento de estos sujetos en relación con la población normal.

En relación a la segunda hipótesis, referida al efecto de los problemas específicos de estos sujetos sobre el rendimiento, nuestras expectativas se ven también confirmadas. Los resultados no permiten rechazar la hipótesis nula de que el aumento del rendimiento es independiente de su afectación motora. De hecho no sólo el grado de afectación motriz parece no influir en la ganancia de ejecución tras un año de escolarización, sino que otros aspectos como el disponer o no disponer de habla inteligible, la edad, el nivel escolar, la propia historia de aprovechamientos escolares e incluso los resultados iniciales en el test de Raven aparecen como no relacionados con el progreso. Estos resultados van en la misma línea de los obtenidos por Eagle (1985), aunque en nuestro caso ni siquiera aparece el modesto papel que la edad mental o el grado de afectación motora tienen sobre los resultados que ella ofrece. Hay que señalar también que tanto las habilidades cognitivas que esta autora estudia (la permanencia del objeto), como la muestra que utiliza (34 niños cuadripléjicos de 9 meses a 12 años) son muy diferentes a las que aquí se han planteado. Sin embargo, sí nos parece importante el recoger la sugerencia que ella hace de que la estimulación cognitiva, física y social que los niños reciben en programas de atención temprana y escolarización pueden compensar o reducir los posibles efectos de la falta de experiencia sensorio-motriz durante la primera infancia. En esta misma linea Meyers, Coleman y Morris (1982) han puesto de manifiesto cómo un programa de entrenamiento en habilidades cognitivas (en este caso diversos tipos de conservación) pueden dar buenos resultados con sujetos de estas características.

Nos queda, entonces, por examinar la influencia de la instrucción sobre el progreso. La tercera de las hipótesis que antes avanzábamos va en esa línea, plantean- do que la justificación del avance pudiere estar en el planteamiento de tareas por parte del profesor algo por encima del nivel de competencia estimado según los rendimientos de los sujetos en el pre-test. Un primer examen de los datos resultado de la observación y que aparecen recogidos en la tabla 6 resulta elocuente; más de un $70 \%$ de las tareas observadas han sido planteadas a un nivel que el alumno ya dominaba a principios de curso, mientras que tan sólo algo más de un $25 \%$ estarían dentro de un nivel de exigencia que podríamos estimar dentro de la zona de desarrollo próximo del sujeto al inicio del curso. Este porcentaje, realmente muy bajo, podría explicar la modesta aunque significativa cuantía de los avances que ofrecen los sujetos.

Un análisis más profundo ha tratado de estimar si el aumento de rendimiento es achacable a la cantidad de tareas planteadas con un nivel de exigencia candidato a ser caracterizado como perteneciente a la zona de desarrollo próximo de cada sujeto. La probabilidad de que el azar sea responsable de las diferencias en rendimiento observadas entre las tareas situadas en la ZDP y en la ZDA es sólo de $13,9 \%$ según nuestros resultados, una cifra por encima del nivel de significación standard $(5 \%)$ para ser considerada como significativa. Sin embargo hay algunos aspectos a tener en cuenta para interpretar este dato. Por una parte este factor es el que alcanza la probabilidad más próxima a la significación de todos los que hemos considerado en nuestro análisis como posibles responsables del avance observado en los alumnos. Por otro lado hay que señalar que el número de observaciones realizadas, aunque importante en su cuantía global, es reducido en relación con el número total de horas lectivas recibidas por cada sujeto a lo largo del curso. En este sentido hay que señalar que la larga huelga de la enseñanza estatal que ocupó una parte importante del curso 1987-88 afectó gravemente no sólo a la enseñanza recibida por los alumnos, sino también a nuestro programa de observaciones en el aula, cosa que, si bien es mucho menos trascendente, sí puede afectar a la interpretación de los resultados que aquí ofrecemos. Por todo ello no creemos ilegítimo dejar cons- 
tancia de la importancia del trabajo en la zona de desarrollo próximo, aunque por ahora no pueda considerarse probado como un factor claramente responsable del avance observado. Los resultados de la continuación de esta investigación a lo largo del curso 1988-89 tal vez permitan arrojar más luz al respecto.

Por lo que se refiere a las características del trabajo realizado en el aula hay que decir en primer lugar que no aparecen diferencias significativas ni entre el modo de trabajar de los tres centros ni entre los diversos profesores participantes en la experiencia, al menos en los aspectos tenidos en cuenta en este trabajo. Hay que hacer constar que el bajo nivel de exigencia de las tareas que se plantean con más frecuencia hace que las características propias del trabajo en el aula queden bastante sesgadas y ofrezcan una información de no mucha riqueza. El hecho de que la tarea se adecúe al objetivo educativo explícitamente pretendido por el profesor y que el niño haga un buen análisis de la tarea en la mayoría de los casos no es nada sorprendente ya que las tareas que se plantean en la mayor parte de los casos implican habilidades que ya tienen perfectamente dominadas. Tampoco resulta sorprendente que las tareas muy difíciles sean peor ejecutadas y las muy fáciles bien ejecutadas. Lo que sí resulta revelador es que la comparación entre la ejecución en tareas ya dominadas y en otras que implican una exigencia de poner en marcha destrezas algo por encima de los ya dominadas por el sujeto muestre que no existen diferencias en el nivel de ejecución entre estos dos tipos de tareas. Si a ello añadimos que todas las observaciones han recogido la realización de las tareas escolares en diada, ya sea maestro-niño o entre iguales, esto implica que el rendimiento de los sujetos no disminuye de un modo significativo cuando se les plantean tareas situadas en la zona de desarrollo próximo.

El hallazgo que consideramos más importante de este trabajo es la caracterización que puede hacerse del modo de trabajo efectivo en la zona de desarrollo próximo a partir de los datos observados. Nuestros resultados señalan claramente un conjunto de peculiaridades que distinguen el comportamiento espontáneo de los pro- fesores cuando se plantean tareas dentro de la zona de cuando estas tareas están ya dominadas por el sujeto. Los profesores emiten un mayor número de mensajes motivacionales, utilizan mejores sistemas de incentivos, dan más refuerzos verbales y sociales, utilizan más comparaciones consigo mismo y hacen más atribuciones internas y controlables, al mismo tiempo que ofrecen mayores ayudas a la ejecución, en forma de mensajes de modelado y de preguntas e instrucciones durante la ejecución en el caso de las tareas planteadas en la ZDP que en los otros casos. Parece, por tanto, que se produce un cambio cualitativo importante en los procesos de comunicación que acompañan a la actividad realizada en diada en la zona de desarrollo próximo y que produce un rendimiento equivalente al obtenido en tareas ya dominadas. Si consideramos estos datos en relación con la probabilidad antes mencionada de que el trabajo en la zona sea en parte responsable del avance de estos sujetos podríamos inferir que un tratamiento educativo en donde se aumentara la frecuencia de tareas planteadas en la zona y que utilizara con los alumnos las estrategias que acabamos de señalar podría obtener unos resultados bastante mejores que los que aquí presentamos.

Varios son los aspectos que merecen ser discutidos en relación con lo que acabamos de exponer. En primer lugar el hecho de que en las tareas planteadas en la zona aparezcan formando un todo conductas de los profesores que están relacionadas con aspectos atributivos y motivacionales, por un lado, y cognitivos y de aprendizaje por otro. Esto es algo que ya ha sido señalado por diversos autores incluidos en la corriente socio-histórica (p. e., Wertsch, 1981; Scribner, 1984), quienes han señado que el concepto de actividad ya expuesto por Vigotsky, y luego desarrollado por Leontiev $(1978,1979)$ permite conjugar en la misma unidad de análisis aspectos pertenecientes a tradiciones investigadoras y teóricas tan dispares como son los referentes a la motivación y a la cognición. En segundo lugar, la presencia de preguntas, instrucciones verbales y conductas de modelado durante el proceso de instrucción son coherentes con las propuestas que el grupo de Illinois (Campione, Brown y 
Ferrara, 1982) realiza respecto al desarrollo de sistemas de entrenamiento metacognitivo para el análisis y resolución de tareas que permita un avance en el rendimiento de sujetos con problemas de aprendizaje. Por último, la presencia de mensajes motivacionales, sistemas de incentivos y atribuciones internas y controlables sobre los resultados de la ejecución entronca con la tradición investigadora en el área de la motivación de logro. Los trabajos de Weiner (1986), Elliot y Dweck (1988), Pardo (1988), o Montero (1989) han puesto de manifiesto cómo la motivación de logro está relacionada con las atribuciones que se realicen respecto a las causas del éxito o fracaso, y cómo estas atribuciones que el niño realiza dependen de las que hagan sus profesores. La importancia de las expectativas y atribuciones que hacen padres y educadores sobre el desarrollo y el aprendizaje de los niños ha sido puesta repetidamente de manifiesto entre nosotros por Palacios (1987; Palacios, González y Moreno, 1987) desde una perspectiva muy próxima a la que aquí venimos manteniendo.

Por lo que se refiere al trabajo con el ordenador puede observarse por una parte que un $16 \%$ de las tareas observadas han incluido su uso y, por otra, que no parece haber diferencias apreciables entre el trabajo realizado con ordenador y el resto de las actividades llevadas a cabo en el aula. Hay diversas causas que explican estos resultados. En primer lugar la disponibilidad de «hardware» $y$ «software» adecuados para su uso con los sujetos objeto de estudio. Hay que tener en cuenta que trabajamos con alumnos con graves problemas de movilidad y que en su mayoría no saben leer y escribir. Ello obligó a la adquisición de periféricos adecuados para la interacción con el ordenador (pulsadores que sustituyan al teclado y sintetizadores de voz). Por otra parte no disponíamos de "software" adecuado para los objetivos instruccionales que nos proponíamos, por lo que una parte del equipo de investigación se dedicó al diseño y programación de programas a la medida de los objetivos planteados. Esto lógicamente consume mucho tiempo y recursos, por lo que en el período observado el número de programas disponible ha sido relativamente reducido. Por último, hay que insistir, como ya hicimos en la introducción, que este instrumento es otra de las herramientas de que dispone el profesor, que en este caso permite realizar unas manipulaciones que de otra forma resultarían imposibles para estos niños, pero que es utílizada al nivel y del modo que le parecen oportunos al enseñante en el seno de su programación del trabajo en el aula. En cualquier caso preferimos aplazar la realización de un estudio más en profundidad de este aspecto para cuando dispongamos de un mayor número de datos sobre el uso de un rango de programas más amplio, tras el segundo año de observación al que antes nos hemos referido.

En resumen creemos que los resultados que aquí se ofrecen permiten sostener una caracterización del trabajo que se realiza en la zona de desarrollo próximo y suministra alguna luz sobre su efectividad. Quizá la adecuación del tratamiento instruccional que reciba cada sujeto a través de adaptaciones curriclares individualizadas a la medida de sus necesidades educativas especiales, y que tengan en cuenta algunos de los aspectos que nuestros resultados recogen, permita mejorar los rendimientos de los alumnos.

Creemos que el mantener un nivel de expectativas optimista sobre las posibilidades de progreso de los niños - y en el caso de los alumnos con necesidades educativas especiales aún más - tiene una gran importancia para su aprendizaje y desarrollo. Pensamos que una formación del profesorado en la que se insista sobre las posibilidades de desarrollo y aprendizaje de estos alumnos, que explique estos procesos haciendo recurso a explicaciones controlables por el propio alumno - de forma que éste adquiera patrones atribucionales sobre los resultados de su rendimiento que no sean propios del lenguaje del handicap- y que enseñe a utilizar sistemas de incentivos adecuados, colaborará a que a los alumnos se les planteen tareas que hagan progresar su nivel de conocimientos y aumenten su repertorio de habilidades. Si a ello se uniera el poner de manifiesto que el contexto de la interacción en el aula es un medio para alcanzar metas de aprendizaje compartidas por profesor y alumno dentro de una actividad significativa para 
ambos, al estilo de la que propone el «Laboratory of Comparative Human Cognition" (1982), probablemente nos encontraríamos con que los resultados que ofreciera nuestro sistema educativo mejorarían sustancialmente. Aunque este estudio no tiene pretensiones de generabilidad creemos que los resultados que se ofrecen van en una línea coherente con estas expectativas.

\section{Notas}

1 Este trabajo es resultado de un programa de investigación sobre las posibilidades del uso de los microordenadores para la educación de los deficientes motóricos patrocinado conjuntamente por el Ministerio de Educación y Ciencia, FUNDESCO e IBM España.

2 ETIEDEM, «Equipo de Trabajo e Investigación Educativa sobre la Deficiencia Motórica» está formado, por el area de deficiencia motórica del Centro Nacional de Recursos para la Educación Especial del Ministerio de Educación y Ciencia, con el que colaboran los otros autores. En la investigación que aquí se presenta han participado, bajo la dirección de María de la Cruz García Lorente los investigadores que a continuación se citan: Encarnación Calvo, Beatriz Gracia Avilés, Luis Martín Caro, Paz Ortega, Paloma López, María Angeles Fierro Alonso y María Cruz Pérez López. José A. Domínguez, Aquilino Lousa y Juan Redondo trabajaron como observadores en las aulas. En la producción de los programas de ordenador ha participado también el ITE, bajo la dirección de Elena Veiguela.

3 Una descripción general del programa de investigación y los diferentes tipos de estudios en él previstos puede encontrarse en ETIEDEM (1989).

4 Una descripción pormenorizada del protocolo de observación y de su uso puede encontrarse en Rosa, Montero y ETIEDEM (1990).

\section{Referencias}

Campione, J., Brown, A. y Ferrara, R. (1982). Mental retardation and Intelligence. En R. Sternberg: Handbook of Human Intelligence. Cambridge; Cambridge University Press. Versión española en Paidós, Barcelona, 1987.

EAGLE, R. S. (1985). Deprivation of Early Sensorimotor Experience and Cognition in the Severely Involved Cerebral-Palsied Child. Journal of Autism and Developmental Disorders, 15 (3), 269-283.

ELLIOT, E. S. y DWECK, C. S. (1988). Goals: An approach to motivation and achievement. Journal of Personality and Social Psychology, 54, 5-12.

Equipo de Asesoramiento Psicopadagogico de Tarrasa. Pruebas pedagógicas graduadas para Preescolar y Ciclo Inicial. Madrid: Aprendizaje-Visor, MEC.

ETIEDEM (1989). Instrucción, aprendizaje y desarrollo en niños paralíticos cerebrales de preescolar y ciclo inicial de EGB. Boletín del Instituto de Ciencias de la Educación de la Universidad Autónoma de Madrid.

LabORATORY OF COMPARATIVE Human COGNITION (1982). A Model System for the Study of Learning Difficulties. Quarterly Newsletter of the Laboratory of Comparative Human Cognition, 4 (3), 39-66.

LEONTIEV, A. N. (1959). Los principios del desarrollo mental y el problema del retraso mental. Versión española en Luria, Leontiev y Vygotsky: Psicología y Pedagogia, Madrid, Akal.

Leontiev, A. N. (1978). Activity. Conciousness and Personality. Englewood Cliffs, NJ; Prentice Hall.

LeONTIEV, A. N. (1979). The problem of activity in Psychology. En J. V. Wertsch. The Concept of Activity in Soviet Psychology. Armonk, NY; M. E Sharpe.

LURIA, A. R. (1969). Las funciones psíquicas superiores y su organización cerebral. Moscú, Editorial de la Universidad de Moscú. Versión española editada por Fontanella, Barcelona, 1983.

MARCHESI, A. y MARTín, E. (en prensa). Del lenguje del trastorno a las necesidades educativas especiales. En A. Marchesi, C. Coll y J. Palacios. Dificultades del desarrollo y del aprendizaje escolar.

MEYERS, L. S., COLEMAN, C. L. y MORRIS, L. M. (1982). Conservation training of three cerebral palsied children. Bulletin of the Psychonomic Society, 20 (1), 14-16.

MONTERO, I. (1989). Motivación de logro: Concepto y medida en el ámbito de la enseñanza. Tesis Doctoral no publicada. Universidad Autónoma de Madrid.

PALACIOS, J. (1987). Reflexiones en torno a las implicaciones educativas de la obra de Vygotsky. En M. Siguan (comp.). Actualidad de L. S. Vygotsky. Barceñlona, Anthropos.

PALACIOS, J., GONZÁlEZ y MORENO. (1987). Ideas, Interacción, ambiente educativo y desarrollo: informe preliminar. Infancia y Aprendizaje, 39-40, 159-170.

PARDO, A. (1988). Motivación de logro y enriquecimiento motivacional. Tesis doctoral no publicada. Madrid, Universidad Pontificia de Comillas.

Robinault, I. A. y DenHOFF, E. (1973). The multiple disfunctions called Cerebral Palsy. Medical and Psychological Aspects of Disability, 14.

Rosa, A. (1985). Filosofia del uso del ordenador en el ámbito educativo. En A. Pfeiffer y J. Galván. Informática y Escuela. Madrid, Fundesco. 
RosA, A., MONTERO, I. y ETIEDEM (1990). Un instrumento de observación para el proceso de instrucción-aprendizaje en sujetos con Parálisis cerebral. Comunicaciones al II Congreso del Colegio Oficial de Psicólogos. Area 2: Psicología y Educación. Madrid: Colegio Oficial de Psicólogos.

SCRIBNER, S. (1984). Cognitive Studies of Work. Quarterly Newsletter of the Laboratory of Comparative Human Cognition, 6 (1-2), número doble monográfico.

VYGOTSKY, L.S. (1978). Mind in Society: The development of higher psychological processes. Cambridge, Mass.; Harvard University Press. Versión española titulada El desarrollo de los procesos psicológicos superiores. Barcelona, Grijalbo, 1979.

VYGOTSKY, L. S. (1982a). El significado histórico de la crisis en psicología. En A. R. Luria y M. G. Iaroshevski (eds.). L. S. Vygotsky: Colección de obras, vol. I. Moscú, Pedagogika.

VYGOTSKY, L. S. (1982b). Sobre los sistemas psicológicos. En A. R. Luria y M. G. Iaroshevski (eds.). L. S. Vygotsky: Colección de obras, vol. I, Moscú, Pedagogika.

WEINER, B. (1986). An Attributional Theory of Motivation and Emotion. Nueva York, Springer-Verlag.

Wertsch, J. V. (1981). Trends in Soviet Cognitive Psychology. Storia e Critica della Psicologia, 2 (2), 219-295.

\section{Extended summary}

This paper presents the preliminary results of a research project on the effect of an instructional treatment on the acquisition of school and cognitive skills in a group of preschoolers and first and second grade children with cerebral palsy. The project spans two years and the results given in this paper refer to the first year.

The school curriculum was divided into specific objectives which involved the training of particular skills. An assessment instrument was adapted in order to evaluate the ability of each child in each area of knowledge. Pre-test and post-tests measurements of performance were taken prior to and after an instructional treatment was carried out lasting the whole academic year. The changes in performance were related to observational data gathered through narrative records of classroom activity taken by observers in each classroom. The observational data was later categorized according to a set of behavioral categories related to the instruction and teacher-child interactions. The programming and carrying out of the instruction program was performed by the teachers, though some tools and guidance were offered by the research team.

Results show a mean gain equivalent to $1 / 3$ of the test criteria for one school year's progress, which is important enough for statistical significance. This gain does not seems to be affected by either the range of motor and speech handicaps, or age and number of years of schooling. Intelligence, assessed through Raven's test, was not found to be relevant either.

When the instruction was examined it appeared that about $70 \%$ of the observed school tasks demanded a level of ability similar or below that already mastered by the pupils at the pre-test. While another $5 \%$ were far above the children at their current level. Only $25 \%$ of the tasks were within a level of demand that made them candidates for being considered as within the Zone of Proximal Development of each individual child. When performance was examined no difference appeared between performance in tasks within the Zone of Actual Development and those within the ZPD. Further examination of the data explored whether the level of requirement for the observed tasks might be considered as being responsible for the gain in knowledge. This appears to be the case only if we accept a level of confidence of $15 \%$; although this is too high to be taken as significant, it does show a certain trend in the data which deserves further attention in future research.

The last stage of the data analysis focuses on the characteristics of classroom interactions during instruction. All of tasks were performed in dyads, most of them teacher-pupil; the particular characteristics of the children under study have to be taken 
into account for it helps to explain the great deal of individual attention they received from the teacher. Results show that when tasks have a level of requirement which is within the children's ZPD teachers' behavior differs significantly from those occasions when they are within the ZAD. Specifically, more motivational messages, more social and verbal reinforcements, better systems of incentives, and internal and controllable attributions about the performance are provided. Also, more help is given during performance in the form of modelling messages, questions and directions during the execution of tasks which are within the ZPD. However, no differences were observed between teachers' behavior; the reason for this might be that teachers' behavior changes, more or less spontaneously, under certain conditions of the children's execution of a task.

This characterization of teachers' spontaneous behavior, when school tasks are within the ZPD, seems to be close to the optimal attributional styles to be used in education. Also they are considered to be adequate modes of instruction, as indicated by current theories on motivation for achievement and psychology of instruction. Finally, it has to be said that activity theory offers a suitable framework for relating these two intellectual traditions in a coherent way. 